NASA/CR—2006-214419

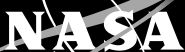

Rate-Based Model Predictive Control of Turbofan Engine Clearance

Jonathan A. DeCastro

QSS Group, Inc., Cleveland, Ohio 


\section{NASA STI Program . . . in Profile}

Since its founding, NASA has been dedicated to the advancement of aeronautics and space science. The NASA Scientific and Technical Information (STI) program plays a key part in helping NASA maintain this important role.

The NASA STI Program operates under the auspices of the Agency Chief Information Officer. It collects, organizes, provides for archiving, and disseminates NASA's STI. The NASA STI program provides access to the NASA Aeronautics and Space Database and its public interface, the NASA Technical Reports Server, thus providing one of the largest collections of aeronautical and space science STI in the world. Results are published in both non-NASA channels and by NASA in the NASA STI Report Series, which includes the following report types:

- TECHNICAL PUBLICATION. Reports of completed research or a major significant phase of research that present the results of NASA programs and include extensive data or theoretical analysis. Includes compilations of significant scientific and technical data and information deemed to be of continuing reference value. NASA counterpart of peer-reviewed formal professional papers but has less stringent limitations on manuscript length and extent of graphic presentations.

- TECHNICAL MEMORANDUM. Scientific and technical findings that are preliminary or of specialized interest, e.g., quick release reports, working papers, and bibliographies that contain minimal annotation. Does not contain extensive analysis.

- CONTRACTOR REPORT. Scientific and technical findings by NASA-sponsored contractors and grantees.
- CONFERENCE PUBLICATION. Collected papers from scientific and technical conferences, symposia, seminars, or other meetings sponsored or cosponsored by NASA.

- SPECIAL PUBLICATION. Scientific, technical, or historical information from NASA programs, projects, and missions, often concerned with subjects having substantial public interest.

- TECHNICAL TRANSLATION. Englishlanguage translations of foreign scientific and technical material pertinent to NASA's mission.

Specialized services also include creating custom thesauri, building customized databases, organizing and publishing research results.

For more information about the NASA STI program, see the following:

- Access the NASA STI program home page at http://www.sti.nasa.gov

- E-mail your question via the Internet to help@sti.nasa.gov

- Fax your question to the NASA STI Help Desk at 301-621-0134

- Telephone the NASA STI Help Desk at 301-621-0390

- Write to:

NASA STI Help Desk

NASA Center for AeroSpace Information 7121 Standard Drive Hanover, MD 21076-1320 
NASA/CR—2006-214419

.

(13.

Rate-Based Model Predictive Control of Turbofan Engine Clearance

Jonathan A. DeCastro

QSS Group, Inc., Cleveland, Ohio

Prepared for the

42nd Joint Propulsion Conference and Exhibit

cosponsored by the AIAA, ASME, SAE, and ASEE

Sacramento, California, July 9-12, 2006

Prepared under Contract NAS3-00145

National Aeronautics and

Space Administration

Glenn Research Center

Cleveland, Ohio 44135 


\section{Acknowledgments}

This work was supported by the Propulsion 21 program at the NASA Glenn Research Center. The Jonathan A. DeCastro gratefully acknowledges the insight and input provided by Kevin Melcher.

This report is a formal draft or working paper, intended to solicit comments and ideas from a technical peer group.

This report contains preliminary findings, subject to revision as analysis proceeds.

Trade names and trademarks are used in this report for identification only. Their usage does not constitute an official endorsement, either expressed or implied, by the National Aeronautics and Space Administration.

Level of Review: This material has been technically reviewed by expert reviewer(s).

Available from

NASA Center for Aerospace Information 7121 Standard Drive

Hanover, MD 21076-1320
National Technical Information Service 5285 Port Royal Road Springfield, VA 22161 


\title{
Rate-Based Model Predictive Control of Turbofan Engine Clearance
}

\author{
Jonathan A. DeCastro \\ QSS Group, Inc. \\ Cleveland, Ohio 44135
}

\begin{abstract}
An innovative model predictive control strategy is developed for control of nonlinear aircraft propulsion systems and sub-systems. At the heart of the controller is a rate-based linear parameter-varying model that propagates the state derivatives across the prediction horizon, extending prediction fidelity to transient regimes where conventional models begin to lose validity. The new control law is applied to a demanding active clearance control application, where the objectives are to tightly regulate blade tip clearances and also anticipate and avoid detrimental blade-shroud rub occurrences by optimally maintaining a predefined minimum clearance. Simulation results verify that the rate-based controller is capable of satisfying the objectives during realistic flight scenarios where both a conventional Jacobian-based model predictive control law and an unconstrained linearquadratic optimal controller are incapable of doing so. The controller is evaluated using a variety of different actuators, illustrating the efficacy and versatility of the control approach. It is concluded that the new strategy has promise for this and other nonlinear aerospace applications that place high importance on the attainment of control objectives during transient regimes.
\end{abstract}

\section{Introduction}

A LTHOUGH model predictive control (MPC) has been used for decades in some form or another in industrial processes, it is gaining ever more acceptance in aircraft propulsion applications due to advances in computing power of modern on-board control platforms. ${ }^{1,2}$ Fast computation is necessary because the controller must generate current and future actuator commands based on open-loop receding horizon trajectories at each time instant. ${ }^{3}$ The trajectory information allows the controller to optimize over the horizon while avoiding a pre-determined set of constraints, such as saturation limits of an actuator or operational limits that define the safety margins of a particular plant or process. In specific propulsion applications such as aircraft engine control or active turbine tip clearance control, this control strategy offers a wide range of utility.

Figure 1 shows a general conceptual sketch of an active clearance control system, where the actuated shroud is depicted as responding to changes in clearance measured by a clearance probe. It is envisaged that replacement of open-loop clearance control systems aboard modern aircraft engines with faster actively-controlled systems will result in ultra tight clearances in the high-pressure turbine, permitting lower emissions and higher fuel savings by decreasing specific fuel consumption (SFC) as well as longer life through decreased exhaust gas temperatures (EGT). ${ }^{4}$ Due to the wide variations in component deformations that occur during flight, if the clearance gap is not large enough, the turbine blades may rub against the shroud and permanently damage the blade tips. These variations arise due to the mismatch in thermal deformations and deformation rates of the rotor and stator assemblies along with centrifugal expansion and contraction of the rotor itself. However, on the basis that every 0.01-inch reduction in clearance results in nearly $1 \%$ improvement in efficiency and $10^{\circ} \mathrm{C}$ reduction in peak EGT during transients, ${ }^{5}$ the need to control clearance as tightly as possible becomes abundantly apparent. Certainly, advances in actuation and sensing hardware for active clearance control will allow for fast and accurate positioning of the shroud relative to the blades, ${ }^{6,7}$ but at these tight clearances, detrimental blade rubs may still arise due to the fact that there is no way of imposing mechanical limits on the clearance. The advantage of applying MPC to this application is that an inherent degree of robustness against blade rubs may enable operation with the tightest clearances that the hardware will allow, enabling the maximum operational benefits to be extracted. 


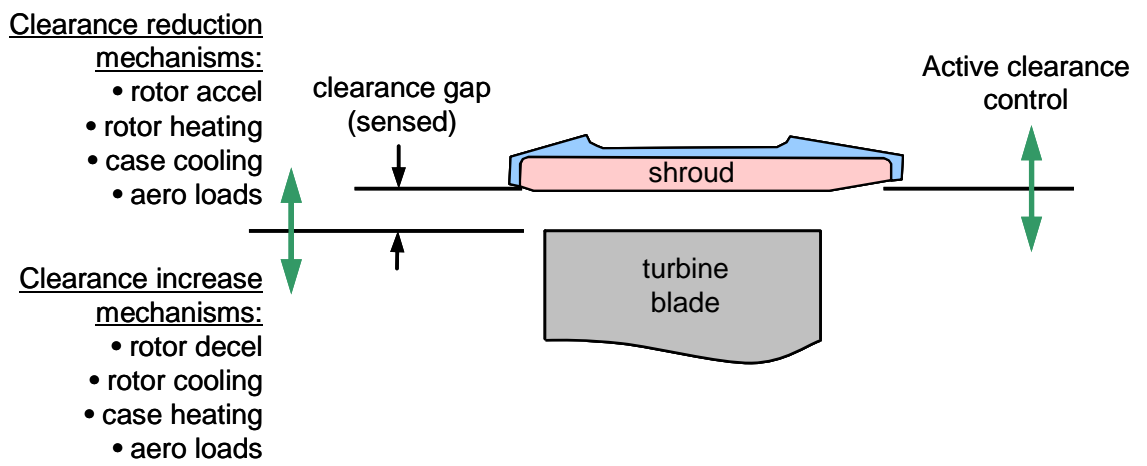

Figure 1. Active turbine clearance control concept (cutaway view of turbine blade tip and shroud).

Because MPC is heavily reliant on an open-loop plant model, the model itself should be an accurate representation of the plant; in this case, a turbofan engine. In numerous cases, the pitfall in applying successful computationally-intense model-based control strategies is in the complexity of the model, as is the case with nonlinear models with a high degree of parameterization (e.g. neural nets). On the other hand, lack of fidelity can result in poor performance or robustness. In this paper, a gain-scheduled, linear parameter varying (LPV) MPC law is considered that retains both computational efficiency and prediction fidelity. In the past, gain-scheduling has often been applied under the assumption that analytical models of the plant exist and are at the disposal of the control engineer, as in Heise and Maciejowski ${ }^{8}$ and Shamma and Cloutier. ${ }^{9}$ Unfortunately, because high-fidelity numerical engine models often used in gain scheduling applications are highly complex, it is nearly impossible to arrive at representative closed-form analytical models. A smaller group of papers, such as Mehra et al, ${ }^{10}$ treat the use of Jacobian linearized LPV models for MPC, but the limitation of traditional LPV models is in the loss in prediction quality when transients cause the models to move away from quasi-equilibrium. Over the horizon, these models may substantially depart from the near-equilibrium regions of the parameter space where model validity holds and substantially compromise optimality. Leveraged by a rate-based linearization method introduced by Leith and Leithead, ${ }^{11}$ an alternate framework conducive to rapidly-varying plants is explored in this work, which operates upon the state derivatives instead of the states themselves. A virtue of this transformation is that fidelity during transients is preserved, enhancing the prediction and global performance of the MPC. To the author's knowledge, this is the first attempt at developing a rate-based LPV modeling technique (hereafter abbreviated as R-LPV) for MPC. The results of the assessment in this paper are useful as further guidelines from which to build MPC controllers for other aerospace applications (e.g. engine control), in addition to the application described here.

This paper is organized as follows. To lay the groundwork for the new MPC, a description of both conventional and rate-based linearization methods for LPV representations as applied to a numerical turbofan engine model are presented. Next, a description of an LPV model predictive controller is given, along with the proposed disturbance model estimation structures. Aspects of applying the MPC to a fast active clearance control device are then described, and details of the actuators used and the high fidelity engine model are given. Finally, results of active clearance control simulations are presented, demonstrating the comparative advantages of the proposed controller over the conventional approach. In that section, both servohydraulic and thermal deformation-based actuators are investigated to illustrate the versatility and robustness of this control approach.

\section{LPV Models of Nonlinear Processes}

The purpose of this section is to introduce concepts relating to linearization of numerical models in the LPV framework, as motivated by the highly nonlinear nature of the turbofan engine. Once these methods are established, germane properties of the two methods are described, as they apply to model predictive control. form:

General nonlinear systems of the form $\dot{x}=f(x, u, v), y=g(x, u, v)$ can be placed in the following standard LPV

$$
\begin{aligned}
& \dot{x}=A(\rho) x+B_{1}(\rho) u+B_{2}(\rho) v \\
& y=C(\rho) x+D_{1}(\rho) u+D_{2}(\rho) v
\end{aligned}
$$


The state vector, control input, disturbance input, and output vector are $x \in R^{n}, u \in R^{n_{u}}, v \in R^{n_{v}}$, and $y \in R^{n_{y}}$, respectively. The scheduling parameter vector is denoted by $\rho$ and can be either exogenous, an input external to the plant, or endogenous, a state of the system. In the latter case, the system is said to be quasi-LPV.

\section{Two Perspectives on Linearization}

Jacobian linearization ${ }^{12}$ is often considered the default method for attaining representations of nonlinear processes, especially those for which analytical expressions do not exist. Although it is possible in theory to linearize away from equilibrium, practical considerations often limit perturbation of the model or actual system to steady-state operating points. This procedure, when conducted at several distinct operating points, produces a family of linear models

$$
\begin{aligned}
& \delta \dot{x}=A(\rho) \delta x+B_{1}(\rho) \delta u+B_{2}(\rho) \delta v \\
& \delta y=C(\rho) \delta x+D_{1}(\rho) \delta u+D_{2}(\rho) \delta v
\end{aligned}
$$

where the perturbation values $\delta x=x-x_{0}, \delta u=u-u_{0}, \delta v=v-v_{0}$, and $\delta y=y-y_{0}$ are functions of the steady-state trim values, $x_{0}, u_{0}, v_{0}$, and $y_{0}$. Although this family can be quite large when adequate coverage of the entire operating envelope is needed, the result is by no means global as the state derivative trim value $\dot{x}_{0}$ must still be assumed to remain zero everywhere. This implies that LPV trajectories are limited to a subset of actual trajectories of the nonlinear system exactly at equilibrium, but this restriction is often relaxed by permitting transitions between points so long as variations in $\rho$ are sufficiently slow. Within the neighborhood of a single linearization point, the LPV input/output relationship is accurate to within first-order of the actual system.

Practically speaking, the family of state-space models is scheduled by either a nearest neighbor or interpolation approach. Since the LPV system in Eq. (2) is actually a nonlinear affine system, trim value updates must be included along with the system matrix updates, as depicted in Fig. 2. If the nonlinearity is particularly dominant, i.e. highly sensitive to minor variations in $\rho$, then quasi-LPV representations may be rendered invalid even with slow variations due to the fact that the trim values are only approximations as the trajectory progresses between operating points. Moreover, the recursive nature of the quasi-LPV parameter update causes any error to accumulate without bound, possibly leading to significant model/plant disparities even within small time frames.

In an alternate linearization perspective known as rate-based linearization, ${ }^{11}$ the nonlinear system is approximated to first order by an affine Taylor series expansion as before, but the states are transformed by simply taking their derivatives. In so doing, the assumed static first-order terms disappear, leaving the actual system state derivatives as the LPV states. Perturbation values now become unnecessary, and the system of equations takes on the following form:

$$
\begin{aligned}
& \ddot{x}=A(\rho) \dot{x}+B_{1}(\rho) \dot{u}+B_{2}(\rho) \dot{v} \\
& \dot{y}=C(\rho) \dot{x}+D_{1}(\rho) \dot{u}+D_{2}(\rho) \dot{v}
\end{aligned}
$$

In this representation, the state vector is now represented by $\xi=\left[\begin{array}{ll}\dot{x}^{T} & y^{T}\end{array}\right]^{T}$ and the state matrices are augmented appropriately. To complete the R-LPV model, initial states must be specified if the model is to start at points other than the origin. Figure 3 shows an input/output signal diagram for this type of model. If the trim values are, in fact, obtained at steady-state, it may be observed that the perturbation states $\delta x$ in Eq. (2) are first-order approximations

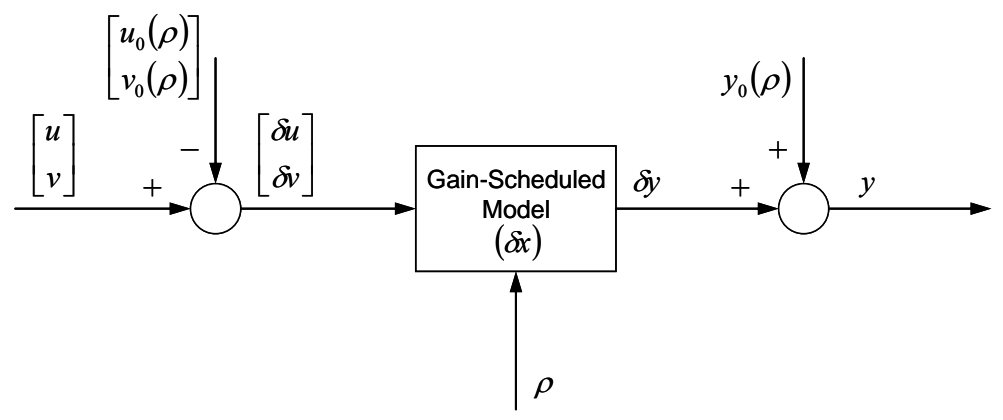

Figure 2. Jacobian LPV models. 


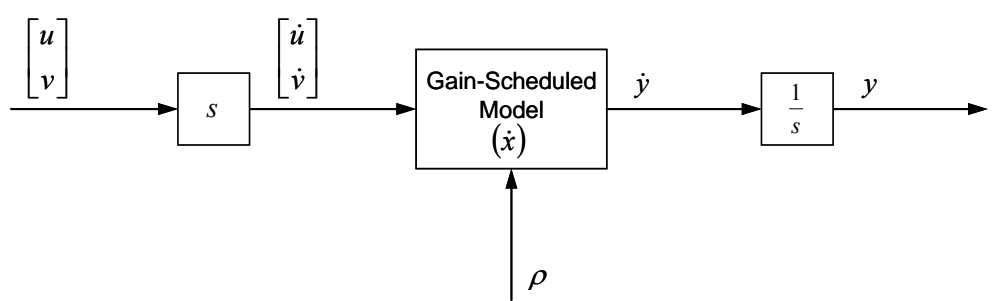

Figure 3. R-LPV models.

to the derivatives of the actual states $\dot{x}$ in the limit sense. The family of Jacobians obtained in Eq. (2) may thus be substituted into Eq. (3) and scheduled identically. In contrast to Eq. (2), however, all trim values including the state derivative trim $\dot{x}_{0}$ become implicit to the model. To elaborate, if $\rho$ is sufficiently slow during a particular transient, then the nonhomogeneous terms in Eq. (2), a linear combination of trim values obtained at equilibrium that are dependent upon $\rho$, will be close enough to reality that the resulting trajectory will closely correspond to the actual. If the system were excited by another transient where $\rho$ is now fast, the nonhomogeneous terms will be incorrectly computed from the subset of trim values at equilibrium, causing inconsistencies between the model and actual trajectories. In the R-LPV setting, these nonhomogeneous terms are assumed constant, resulting in Eq. (3) being linear. No restrictions are therefore imposed on these terms being constructed from equilibrium trim values.

R-LPV models have improved fidelity at all neighboring points in the parameter space, including nonequilibrium conditions, implying that the slow variation condition may be relaxed to some extent. Furthermore, by removing the state derivative trim and all remaining trim values from the formulation, these quantities no longer need to be approximated. This does not solve the problem of obtaining new Jacobians away from equilibrium, but the reduced parameterization and interpolation thereof lessens propagation of error across the trajectory existent in quasi-LPV formulations. In the context of receding horizon control, improved fidelity means that global optimality is more closely preserved. Because the open-loop model is effectively a quasi-LPV model that recursively executes several samples into the future, reduced error propagation becomes equally important in realizing global optimality as maintaining fidelity in non-equilibrium regimes.

The penalty for the improved model is that the state vector dimension is now expanded to $n+n_{y}$ and that the computed control signal is the integral of the actual controller output, $u=\int u^{\prime}$, due to the rate-dependency of the controller structure. Conversely, the measured disturbance inputs to the LPV models are brought in as a derivative, i.e. $v^{\prime}=d v / d t$. It should be cautioned that introduction of uncertainties may certainly have a deleterious effect due to this derivative expression if these inputs are susceptible to noise or biasing, especially in plants with high inputoutput sensitivities. In practice, this disturbance is often manifested as a demand-related event that is subject to little noise or other types of uncertainty. Moreover, estimation structures that operate upon separate disturbance states decouple the estimation loop from these effects.

\section{Model Predictive Controller in Rate-Based LPV Form}

In this section, the practical problem setup for MPC is given. First, the disturbance model structure for output estimation in rate-based form is presented with treatment given to consolidating the estimator states with the expanded R-LPV states. Then, with this complete plant/estimator model, the R-LPV formulation of MPC is provided.

\section{Disturbance Models in the Rate-Based Paradigm}

In receding horizon control, input/output disturbance models are necessary to reduce the effects of any plantmodel mismatch and to absorb exogenous disturbances affecting the plant but unknown to the model. Due to lack of information regarding most disturbances, such models are often chosen to be pure integrators so that any constant disturbance may be asymptotically rejected and output tracking may theoretically occur without error. It is well known that, if the augmented system is detectable and the number of disturbance states equals the number of outputs, then all output channels will have zero steady-state error. ${ }^{13,14}$ Normally, the state vector is expanded to incorporate these new disturbance states, $d \in R^{n_{y}}$. However, this approach can be improved to avoid undue computational complexity arising from incorporating an additional $n_{y}$ integrator states (a total of $2 n_{y}$ more than the original model). Recognizing that the output integration is decoupled from the plant dynamics, the disturbance 
model inputs are simply added to $\dot{y}$ in Fig. 3 and the state sub-vector $y$ in Eq. (3) now becomes $y+d$. The state space equations are then

$$
\begin{aligned}
& \dot{\xi}=\bar{A} \xi+\bar{B}_{1} u^{\prime}+\bar{B}_{2} v^{\prime}+\bar{B}_{3} w_{p} \\
& y=\bar{C} \xi+w_{m}
\end{aligned}
$$

with the augmented matrices

$$
\bar{A}=\left[\begin{array}{cc}
A(\rho) & 0 \\
C(\rho) & \Lambda_{n_{y}}
\end{array}\right], \quad \bar{B}_{1}=\left[\begin{array}{l}
B_{1}(\rho) \\
D_{1}(\rho)
\end{array}\right], \quad \bar{B}_{2}=\left[\begin{array}{c}
B_{2}(\rho) \\
D_{2}(\rho)
\end{array}\right], \quad \bar{B}_{3}=\left[\begin{array}{c}
0 \\
I_{n_{y}}
\end{array}\right], \quad \bar{C}=\left[\begin{array}{ll}
0 & I_{n_{y}}
\end{array}\right]
$$

As in Eq. (3), the augmented state vector $\xi$ is composed of the state derivative vector and the output vector. Uncorrelated additive process and measurement noise intensities, $w_{p} \in R^{n_{y}}$ and $w_{m} \in R^{n_{y}}$, enter the disturbance model input channels and measurement channels, respectively. The diagonal filter matrix $\Lambda_{n_{y}}$ is included to avoid pure integration at the outputs. Note that the R-LPV realization augmented with disturbance models will have the same number of augmented states as conventional LPV realizations with disturbance models.

\section{Model Predictive Control Law}

Given the R-LPV plant/estimator model, optimal control actions are found by minimization of a finite-horizon linear quadratic objective function with respect to the controls and the outputs. ${ }^{3}$ Converting to discrete time, the linear-quadratic cost function for the MPC law may be written as

$$
J_{k}=\sum_{i=1}^{p}\left[\left(y_{k+i}-y_{r, k+i}\right)^{T} Q\left(y_{k+i}-y_{r, k+i}\right)+u_{k+i}^{T} R u_{k+i}\right]
$$

subject to the discretized version of the plant model of Eq. (4) and constraints on the manipulated controls and measured outputs at time $k$. The subscript $r$ denotes the reference setting and $p$ represents the prediction horizon. The symmetric, positive-definite weight matrices $Q$ and $R$ are to-be-determined design parameters, and the usual requirement holds that the pairs $\left(\bar{A}, \bar{B}_{1}\right)$ and $\left(\bar{A}, Q^{1 / 2}\right)$ are stabilizable. A Kalman filter state estimator is designed on the condition that $(\bar{C}, \bar{A})$ is detectable, assuming the process and measurement noise covariances $W$ and $V$, defined by the intensities $w_{p}$ and $w_{m}$, respectively. It is important to note that, in order to impose any weighting or constraints on the actual controls in the rate-based MPC framework, the output vector must be augmented with those control inputs.

The LPV MPC formulation is achieved by computing state prediction equations recursively at each time step. Using the same state space notation from continuous time for the discrete time system equations, the state predictions are computed by

$$
\left[\begin{array}{c}
\hat{\xi}_{k+1} \\
\vdots \\
\hat{\xi}_{k+p}
\end{array}\right]=\mathbf{A} \hat{\xi}_{k}+\mathbf{B}_{1} u_{k-1}^{\prime}+\mathbf{B}_{1}^{\Delta}\left[\begin{array}{c}
\Delta u_{k}^{\prime} \\
\vdots \\
\Delta u_{k+p}^{\prime}
\end{array}\right]+\mathbf{B}_{2}\left[\begin{array}{c}
v_{k}^{\prime} \\
\vdots \\
v_{k+p-1}^{\prime}
\end{array}\right]
$$

where $\hat{\xi}_{k}$ are the estimated R-LPV states. The prediction matrices are

$$
\mathbf{A}=\left[\begin{array}{c}
\bar{A}(k) \\
\vdots \\
\prod_{i=0}^{p} \bar{A}(k+i)
\end{array}\right], \quad \mathbf{B}_{1}=\left[\begin{array}{c}
\bar{B}_{1}(k) \\
\vdots \\
\sum_{j=1}^{p-1} F_{1}(j, k+p)+\bar{B}_{1}(k+p-1)
\end{array}\right],
$$




$$
\begin{gathered}
\mathbf{B}_{1}^{\Delta}=\left[\begin{array}{cccc}
\bar{B}_{1}(k) & 0 & \cdots & 0 \\
\vdots & \vdots & \ddots & \cdots \\
\sum_{j=1}^{p-1} F_{1}(j, k+p)+\bar{B}_{1}(k+p-1) & \sum_{j=1}^{p-2} F_{1}(j, k+p)+\bar{B}_{1}(k+p-1) & \cdots & \bar{B}_{1}(k+p-1)
\end{array}\right], \\
\mathbf{B}_{2}=\left[\begin{array}{cccc}
\bar{B}_{2}(k) & 0 & \cdots & 0 \\
\vdots & \vdots & \ddots & \cdots \\
F_{2}(p-1, k+p) & F_{2}(p-2, k+p) & \cdots & F_{2}(1, k+p)
\end{array}\right],
\end{gathered}
$$

where

$$
F_{*}(j, \ell)=\left[\prod_{i=1}^{j} \bar{A}(\ell-i)\right] \bar{B}_{*}(\ell-j-1)
$$

In this representation, the control inputs are split into the present value of the control derivative $u_{k}^{\prime}$ and a vector of future control derivative moves.

The trajectory of predicted outputs is

$$
\hat{y}_{k+i}=\bar{C}(k+i) \xi_{k+i}+D_{2}(k+i) v_{k+i}^{\prime}
$$

for each $i=1, \ldots, p$. The dependence of the Jacobians on $\rho$ is implied by their discrete-time dependence on $k$. From the above equations, it is readily apparent that the internal models assume quasi-LPV formulations (an internally-computed scheduler) in forming the predictions, but may use an external scheduler for state estimation as long as state continuity between each realization is guaranteed. Because the internal model must be strictly proper; i.e. zero gain at infinite frequency, it is necessary to formulate Jacobian LPV systems without the $D_{1}$ term. In contrast, the fundamental model in R-LPV systems may be of any type because the R-LPV transformation is, by definition, one that maps any arbitrary system to a strictly proper system because the original $D$ matrices become part of the $\bar{B}$ matrices.

Standard quadratic programming $(\mathrm{QP})$ approaches are available to efficiently solve this convex constrained optimization problem; the approach employed here uses an "active set" method that optimizes on a subset of inequality constraints that are active at the current step. Common ways of making the control law computation more efficient are to limit the control horizon $m$ to some $m<p$ or to update only intermittently (known as blocking). To expand the feasible set of the QP problem, the constraints are not posed as hard limits but instead are softened by incorporating a slack variable on the cost function that introduces an expensive penalty should any constraint violations occur. Computation is further streamlined by generating the Jacobians, estimator gains, and prediction matrices in an offline batch mode as a set of parsed arrays so that the on-line algorithm only needs to perform linear interpolation on these arrays at each time step.

\section{Active Tip Clearance Control in a Commercial Turbofan Engine}

As depicted in Fig. 1, the clearance control actuator is designed to move a segmented shroud structure toward and away from the turbine blades. The concept, as referred to in Lattime, et al $l^{15}$ consists of several identical actuators that are placed at locations around the shroud circumference with each effecting movement in one shroud segment. It is assumed that each actuation point has a collocated clearance measurement at the shroud's frame of reference. In this configuration, the detected clearance is the difference between the combined deformation of turbine components (referred to as the un-actuated clearance) and the actuator displacement, where a positive displacement results in smaller clearance. The active clearance control system can operate either upon a minimum detected clearance and single command signal, or upon several local clearance signals and multiple actuator commands. The MPC is adaptable to both configurations, but only the single-output case is considered here. 
Two actuator platforms were used to demonstrate predictive control behavior across different realistic hardware design scenarios: one representing a servohydraulic actuator and the other representing a thermal deformation-based actuator. A detailed nonlinear model of the servohydraulic actuator described in DeCastro and Melcher ${ }^{16}$ is implemented as the truth model and a parsimonious reduced order model is used in the MPC. The dynamics of the actuator are primarily driven by the servo control loop and are approximated here by the following third-order system with corner frequency of approximately $0.2 \mathrm{~Hz}$ :

$$
G=\frac{1283}{(s+223.2)(s+4.035)(s+1.424)}
$$

The actuator stroke is measured by a transducer for servo-control and is therefore accessible to the engine control unit as a measured state of the system. The second actuator, a thermal-deformation based device, is similar to current thermal actuation systems that utilize secondary engine air to regulate the flange attachment to the shroud structure by way of a proportional air valve. ${ }^{4}$ This device is represented by the first order system

$$
G=\frac{1}{\tau s+1}
$$

Because the intent of this case is purely illustrative, the thermal actuator internal model and truth model are taken to be identical. For both actuator types, constraints were placed on the minimum and maximum allowable control input settings, corresponding to zero and 0.1 -inch displacements, respectively.

For this tip clearance application, a commercial engine simulation (CES) representative of a large, high-bypass turbofan engine coupled with the actuator model was used as the baseline truth model for closed-loop evaluation. The simulation features a detailed model of the turbine section component deflection to accurately represent demand-related clearance effects. On the basis that the MPC is to be implemented as an isolated control loop from the engine controller, a simplified closed-loop engine fan speed compensator was incorporated. The compensator is a linear, second order system with a free integrator at the output, designed to obtain a slightly overdamped nominal closed-loop bandwidth of approximately $0.6 \mathrm{~Hz}$. The requested fan speed is actually a mapping function consisting of power lever angle (PLA) and three environmental parameters (altitude, Mach number, ambient temperature). Multi-mode controllers that typically deal with limits normally encountered with large transients have not been incorporated, but the MPC structure can be viably altered in practice to accommodate this additional level of sophistication. Alternately, if the tip clearance MPC is implemented in a unified engine MPC, separate models of the controller are unnecessary because the MPC generates these engine commands.

Nine linear models were generated of the closed-loop engine at equispaced PLAs between ground idle and maximum power at sea-level static conditions, constructed with several sensed output channels and two input channels: delta clearance (provided by the actuator) and fuel flow. Jacobians at these points were found by perturbing these inputs, nine engine states (fan and core shaft velocities and seven internal metal temperatures), and the three fan speed controller states. The turbine component states are numerous and were omitted from the linearization. Because the operating conditions considered here are limited to constant altitude and airspeed, the scheduler is taken simply as the measured fan speed, $X N 2$.

With the hydraulic actuator model, the LPV model has a total of two inputs, fifteen states, and six outputs; these I/O's are listed in Table 1. The total number of R-LPV internal model states is $22\left(n+n_{y}+n_{u}\right)$, only one state greater than the Jacobian LPV counterpart. Figure 4 shows the interconnection of the rate-based active clearance control MPC and the turbofan engine plant. The exogenous disturbance $\left(\delta_{k}\right)$ is brought in only to the engine, while the demand-induced measured disturbance $\left(v_{k}\right)$ input, $P L A$, is brought in to the engine and MPC. This may be extended to capture other sensed inputs (ambient temperature, Mach number, altitude) if needed. As mentioned earlier, differentiation of input variables is necessary in the rate-based internal model framework. Such a transformation can amplify any noise or biasing on the input channels, but since this application calls for a disturbance that is a function of throttle position, it may be argued that the signal is mostly free of noise and other sources of uncertainty. Nonetheless, examining the effect that these uncertainties have on transient performance is warranted to ensure that optimality is not compromised. 
Table 1. Inputs and outputs for R-LPV tip clearance controller.

\begin{tabular}{|c|c|}
\hline Inputs & Outputs \\
\hline Actuator command, $U A C T$ & Fan speed, $X N 2$ \\
Power lever angle, $P L A$ & Fuel flow, $W F$ \\
& Exhaust gas temperature, $T 5$ \\
& Stage 1 clearance, $C L R$ \\
& Actuator position, $X A C T$ \\
& Actuator command, $U A C T$ \\
\hline
\end{tabular}

\section{Design Considerations}

In this tip clearance implementation, minimization of SFC and EGT is indirectly achieved by penalizing clearance excursions away from a small-valued set point. Hence, the objective function and loop transfer function reduce to single-input, single-output (SISO), with the actuator command as the control input and clearance as the regulated output. The unconstrained SISO control input is then used exclusively for design:

$$
u_{k}^{\prime}=-K \hat{\xi}_{k}
$$

where $K$ is the full-state feedback gain matrix.

The tuning procedure selected in this paper follows Doyle and Stein, ${ }^{17}$ where the objective is to first specify a desired loop transfer function (closed or open), then apply a quasi-deadbeat estimator to the loop to asymptotically achieve the specification. With the ratio $R / Q$ set to 0.001 , the controller attains fast response but avoids highfrequency gain/phase distortions in the control loop return ratio $K(z I-\bar{A})^{-1} \bar{B}_{1}$ due to discretization effects. In so doing, the return ratio more closely resembles its continuous-time counterpart, which has the property of providing infinite gain margin against gain increases and $60^{\circ}$ of phase margin.

\section{Demonstrative Simulations}

In this section, simulated results of the active clearance control MPC application are given and comparisons made between controllers derived using conventional internal modeling methods and the rate-based method explained in this paper. Additionally, ties are made to the practicality of this MPC implementation in active clearance control using the two actuation methods described earlier. For all reported results, a $50-\mathrm{Hz}$ sample rate was used. The minimum clearance constraint and clearance set point were set to identical values, which presents the

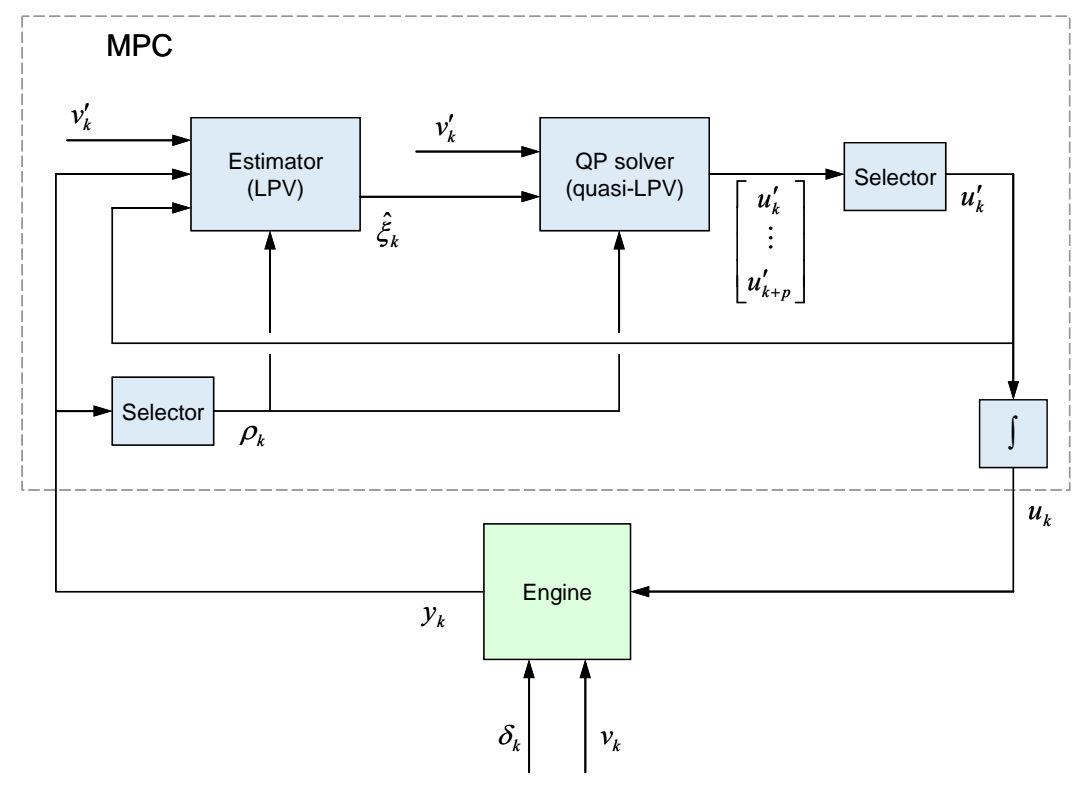

Figure 4. Block diagram of MPC with R-LPV models. 
most demanding scenario for the controller since maintenance of the clearance set point and avoiding blade rubs are now invoked as competing objectives.

\section{Distinctions between Rate-Based and Jacobian MPC}

Clearance responses with the Jacobian-MPC law for the servohydraulic actuator given a large-magnitude PLA transient are shown in Fig. 5. For this assessment, the prediction horizon $p$ was set to 20 samples and the control horizon $m$ to 2 samples, with both these settings chosen to obtain an unconstrained response that resembles the infinite-horizon response. In the figure, fan speed and adjusted clearance (top and bottom traces, respectively) represent measured outputs. While not a physical output, the un-actuated clearance shown in the middle trace represents the combined deformation of the turbine section components, a demand-related effect from the engine essential for predicting the evolution of clearance through time. This quantity is computed as the sum of the controlled clearance and the actuator displacement (not shown), which is a valid estimate only since the feedback effects of clearance on the engine's gas path dynamics are considered to be minor.

In the bottom trace of Fig. 5, it is clear that the transient causes the adjusted clearance to fall below the constraint (dashed line) at about 1.8 seconds, which is in violation of the objective that the adjusted clearance must be kept positive to avoid the possibility of blade rubs. The open-loop prediction trajectory overlays reveal that the model incorrectly predicts the evolution of fan speed and un-actuated clearance outputs, especially during the time interval between 1.8 and 2.2 seconds, which correlates to the time when the constraint violation begins. The output trajectory plot in Fig. 6 is helpful for visualizing gradient information in the output space, containing the same prediction trajectories as Fig. 5 plus one additional trajectory at the 1.8-second mark. As expected, the 1.8-second prediction has the largest initial gradient error of the four trajectories, as shown by the large migration away from the actual trajectory early on in the horizon.

The improvements that the R-LPV models afford the optimization are shown in Fig. 7, given the same PLA transient and an identically-tuned controller (i.e., one with the same design parameters). In comparison with the previous case, the adjusted clearance (bottom trace) stays at or above the constraint, satisfying the blade rub objective. The prediction traces in Fig. 7 and associated output trajectory plot in Fig. 8 show that the sharp, erratic responses disappear, with initial gradients following much closer to the actual. Note that the 1.5 -second prediction traces settle out at constant rates-of-change. The reason for this is because the measured disturbance derivative, not the measured disturbance itself, is held constant throughout the horizon in the R-LPV setting. For all simulations
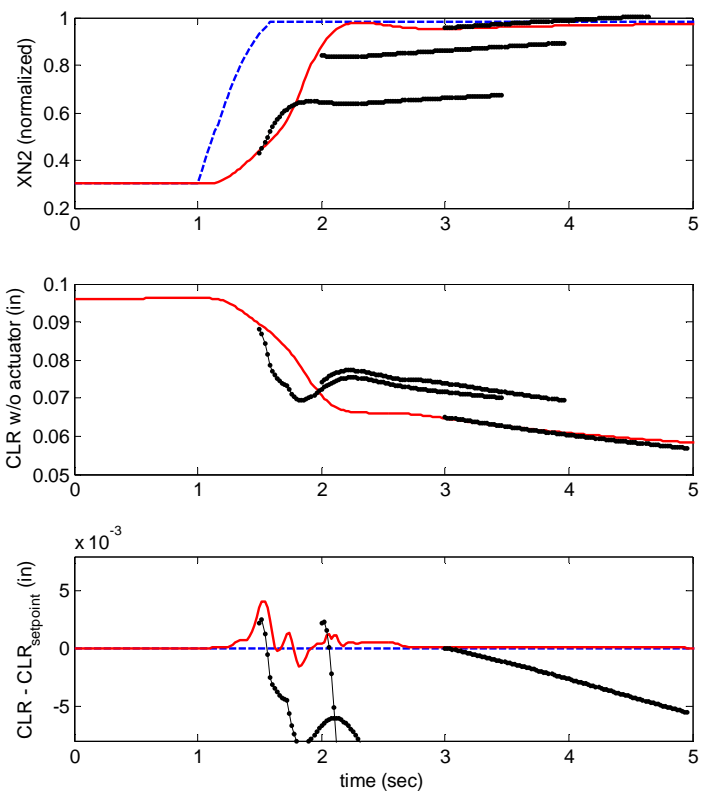

Figure 5. Jacobian LPV responses, showing actual (red) and predicted outputs (black dots).

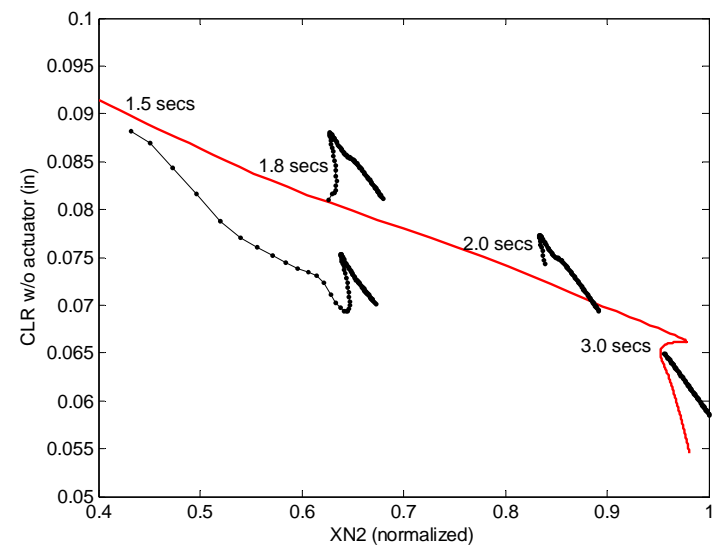

Figure 6. Jacobian LPV output trajectories, showing actual (red) and predicted outputs (black dots). 

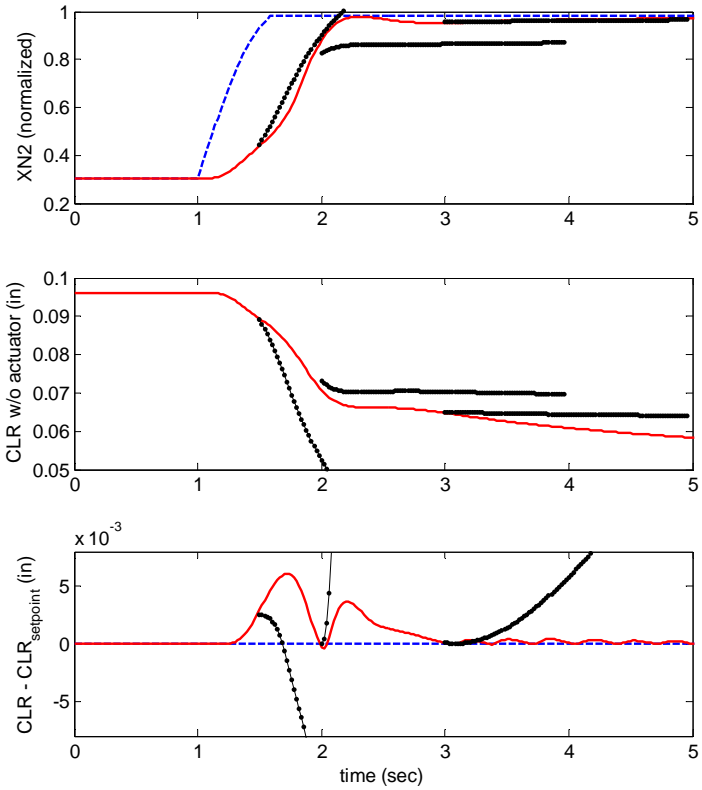

Figure 7. R-LPV responses, showing actual (red) and predicted outputs (black dots).

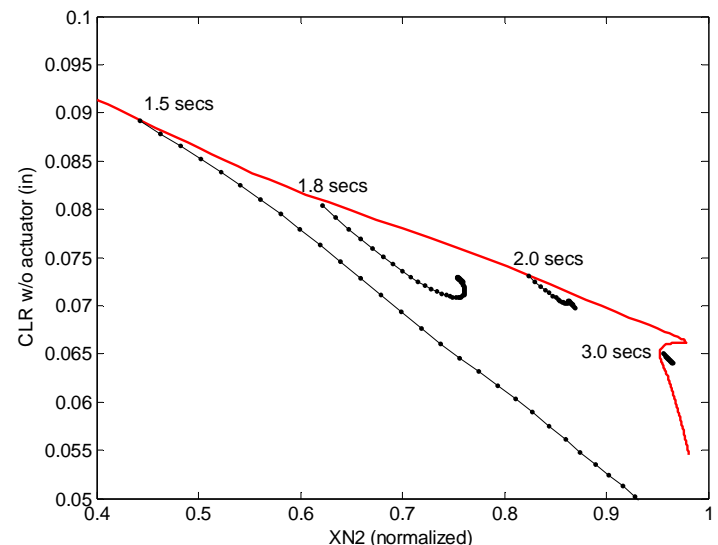

Figure 8. R-LPV output trajectories, showing actual (red) and predicted outputs (black dots).

executed, this does not negatively impact the convergence of the controller. At 3.0 seconds, all internal model transients die out, with any subsequent error being a result of the slow neglected clearance dynamics. The closer agreement between the R-LPV models and the nonlinear plant throughout the transient is owed to the fact that the non-homogeneous terms in the R-LPV models are no longer explicitly parameterized and therefore not subject to the associated limitations.

\section{Practical Assessment of the Rate-Based MPC}

Where the previous evaluation was only concerned with utilizing the feedforward attributes of the MPC in optimizing control actions based on demand-induced clearance events, actual in-flight situations require the need to reject exogenous disturbances and maintain performance with changing parameters, an exercise that is largely a question of feedback compensation. In the active clearance control application, clearance changes induced by flight loads acting on the engine are considered, whose effects become more problematic as clearance set points decrease because of the decreased margins against blade rubs. As alluded to in Olsson and Martin, ${ }^{18}$ there are multitudes of engine transients that occur during flight that are of interest in active clearance control; a sampling of which includes takeoff, re-burst, thrust reversal, hard turns, and aircraft stall. Each event contributes a demand-induced component on clearance due to PLA, etc., and a presumed unknown flight load-induced component as a result of turbulence, maneuvering of the aircraft, and asymmetric thrust loads that cause the engine to bend. Because of their large, rapidly-acting contributions on clearance, the takeoff, thrust reversal, and aircraft stall events are employed to exercise the MPC laws given realistic clearance events that occur during normal operation; the magnitudes and rates-of-change of each event were reproduced approximately from actual flight data reported in Ref 18 . Since takeoff and re-burst are essentially identical in nature aside from the thermal state of the turbine at the start of the event, examining the transient MPC response with a takeoff event effectively serves the dual purpose.

To compare the MPC strategy with a controller very similar to a linear quadratic Gaussian (LQG) control law, the clearance constraint was removed from the MPC computations, rendering the new controller to be similar by nature to a gain-scheduled LQ controller with anti-windup protection. The purpose of this comparison is to justify the benefit of MPC in the face of the additional computational burden. Note that the unconstrained, LQG-like controller is still globally valid in the sense of Eq. (3). If a truly gain-scheduled LQG controller were used, this would be optimal only in the vicinity of the linearization points. Figure 9 shows the MPC response given the three event scenarios at nominal conditions for a thermal actuator with 1.6-second time constant $(\tau=1.6)$. This case represents an actuator whose response rate roughly matches the maximum deformation rate of the rotor during a 

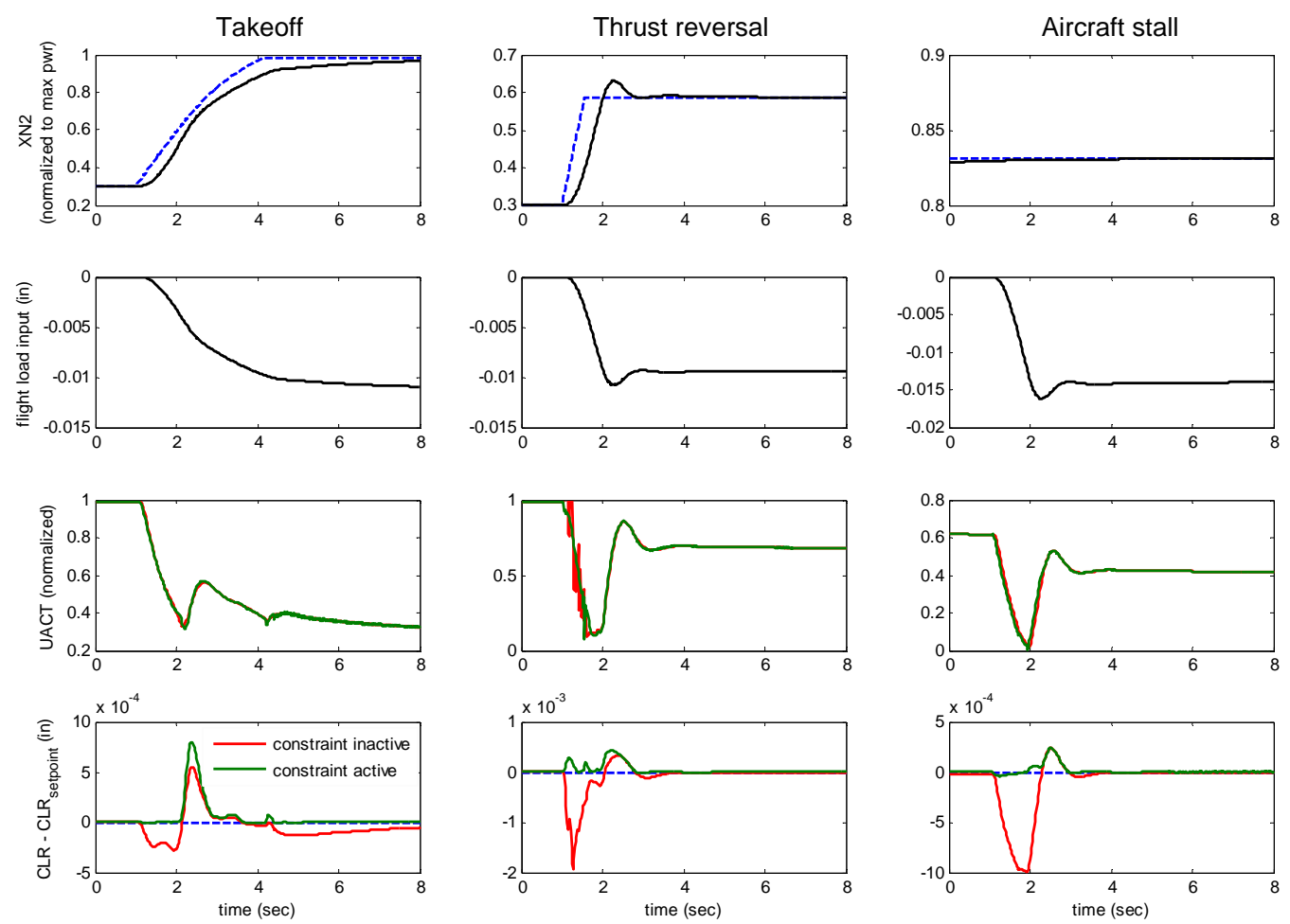

Figure 9. R-LPV responses for fast thermal actuator.

power-increase event. To extend the anticipation capability, the prediction horizon $p$ was increased to 50 samples and control horizon $m$ to 5 samples. From the figure, the MPC response to a thrust reversal transient produces the most benefit over an unconstrained controller, eliminating the 2-mil excursion below the constraint. It is encouraging that enforcement of the clearance constraint is a result of very smooth actuator command signals rather than abrupt or highly oscillatory control actions. Although unconstrained responses in the remaining two events have milder constraint violations, it is important to note that the constrained controller satisfies the blade rub objective in both cases. One may interpret these results from a systems point-of-view. If the clearance setpoint using this LQG-like controller must be 2 mils greater than the MPC setpoint to accommodate the possibility of the worst-case event, then this would detract from exploiting the benefits of a tip clearance control system. Based upon past studies, ${ }^{5}$ the penalty on efficiency would be $0.2 \%$ and the penalty on EGT overshoot would be $2^{\circ} \mathrm{C}$.

Figure 10 shows the response with a thermal actuator with $\tau=5.3$, a rate $60 \%$ slower than the previous case.
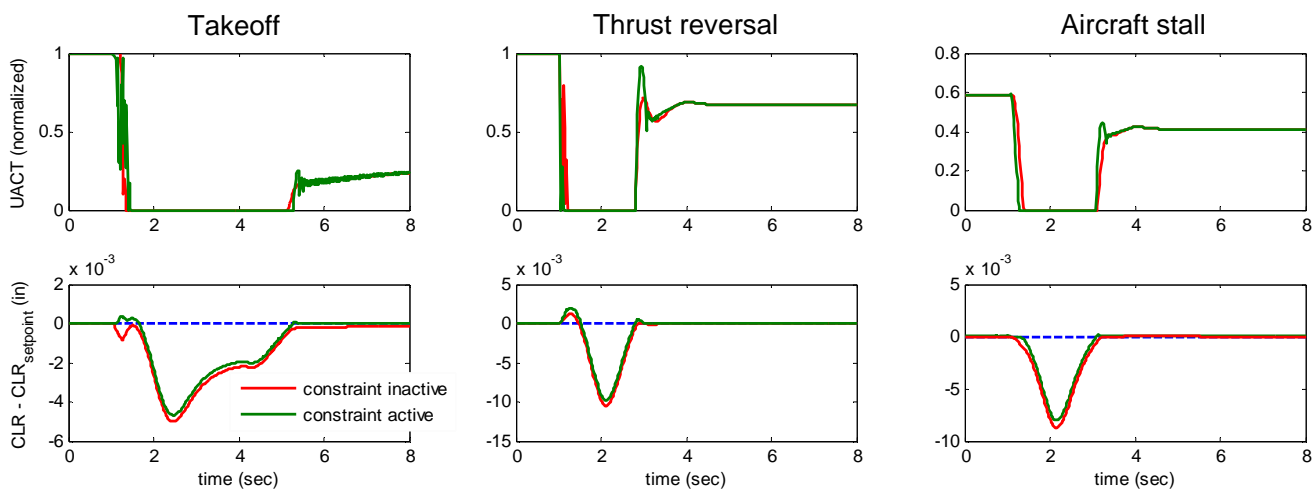

Figure 10. R-LPV responses for slow thermal actuator. 
Table 2. Minimum clearance for constrained and unconstrained R-LPV MPC. Values are in mils

\begin{tabular}{|c|c|c|c|}
\hline \multicolumn{4}{|c|}{$\left(1 \times 10^{-3}\right.$ in $)$. } \\
\hline & Unconstrained & Constrained & Delta \\
\hline \multicolumn{4}{|c|}{ Thermal Actuator, $\tau=1.6$, nominal engine } \\
\hline Takeoff & -0.28 & -0.01 & 0.27 \\
\hline Thrust reverse & -1.93 & -0.01 & 1.92 \\
\hline Aircraft stall & -1.00 & -0.04 & 0.96 \\
\hline \multicolumn{4}{|c|}{ Thermal Actuator, $\tau=5.3$, nominal engine } \\
\hline Takeoff & -5.01 & -4.69 & 0.32 \\
\hline Thrust reverse & -10.5 & -9.80 & 0.70 \\
\hline Aircraft stall & -8.71 & -8.02 & 0.69 \\
\hline \multicolumn{4}{|c|}{ Hydraulic Actuator, nominal engine } \\
\hline Takeoff & -0.80 & -0.18 & 0.62 \\
\hline Thrust reverse & -2.29 & -0.50 & 1.79 \\
\hline Aircraft stall & -3.26 & -0.71 & 2.55 \\
\hline \multicolumn{4}{|c|}{ Hydraulic Actuator, off-nominal engine } \\
\hline Takeoff & -0.60 & -0.13 & 0.47 \\
\hline Thrust reverse & -2.15 & -0.50 & 1.65 \\
\hline Aircraft stall & -3.26 & -0.70 & 2.56 \\
\hline
\end{tabular}

This case is included to illustrate the consequences of applying MPC to an actuator that is slower than the rate of mechanical rotor growth, similar to state-of-the-art actuators. In the figure, the range of the control signal lies between one and zero and saturates beyond those values. For both controllers, the control signal saturates at zero in attempt to move the shroud away from the blades. With the constrained controller, anticipation of the event in the takeoff and thrust reverse events results in a slight increase in adjusted clearance before becoming altogether negative upon loss of authority. Although no anticipation is possible in the purely exogenous stall event, the adjusted clearance stays at zero longer than for the unconstrained case, a result of the more rapid compensation of the constrained MPC when riding the clearance constraint. It may be suggested that MPC performance can be further improved with anticipation of the exogenous events, perhaps either by direct estimation of the flight load event or by correlating to other measurements (e.g. structural sensors). These effects may not be assumed uniform around the turbine circumference.

Table 2 summarizes the MPC benefits for the three actuators and also presents a case of tip clearance control amidst off-nominal engine conditions. Note that any violations of the constraint are a result of the slack tuning parameter described earlier. Comparing the fast thermal actuator with the nominal hydraulic device, both the unconstrained and constrained clearance values are quite different, which is directly related to sub-optimality introduced with plant/model mismatch. Recall that the nonlinear hydraulic actuator is adopted as the truth model, while the thermal actuator only uses the linear model. With the exception of the slow thermal device, enforcement of the constraint results in commensurate amounts of clearance improvements for each. As echoed in Fig. 10, the MPC applied to the slow actuator offers some improvement, albeit less than a full-authority actuator does. The degraded engine case is implemented as a shift in engine parameters to roughly represent 6000 cycles of engine service life degradation, as described in Sallee, ${ }^{19}$ coupled with a shift in fuel flow sensitivity of $-5 \%$. In a gross dynamical sense, any engine degradation is not expected to significantly impact the behavior of the engine, and much less the behavior of clearance dynamics. This important attribute is exemplified by the results.

To address the aforementioned issue concerning the integrity of the internal model amidst uncertainties in the measured disturbance signal, simulation cases were generated where the PLA measured disturbance input was fouled by uncertainties. The results are shown in Fig. 11, where two cases show the effects of the MPC receiving PLA commands corrupted by a $\pm 5 \%$ shift in sensitivity and a third where the $P L A$ has additive white noise with variance of approximately $6 \%$ of full scale. The traces show that the introduction of noise causes excitation of higherfrequency controller modes, but no adverse effects in control performance. Although the controller sees a PLA derivative that differs greatly from the actual value entering the engine, the deadbeat estimator is quick to reject these disparities and the prediction is largely unaffected during the comparatively short span of the horizon. Naturally, longer horizons will exacerbate the effects of noise on the input channel, possibly producing an erratic or 

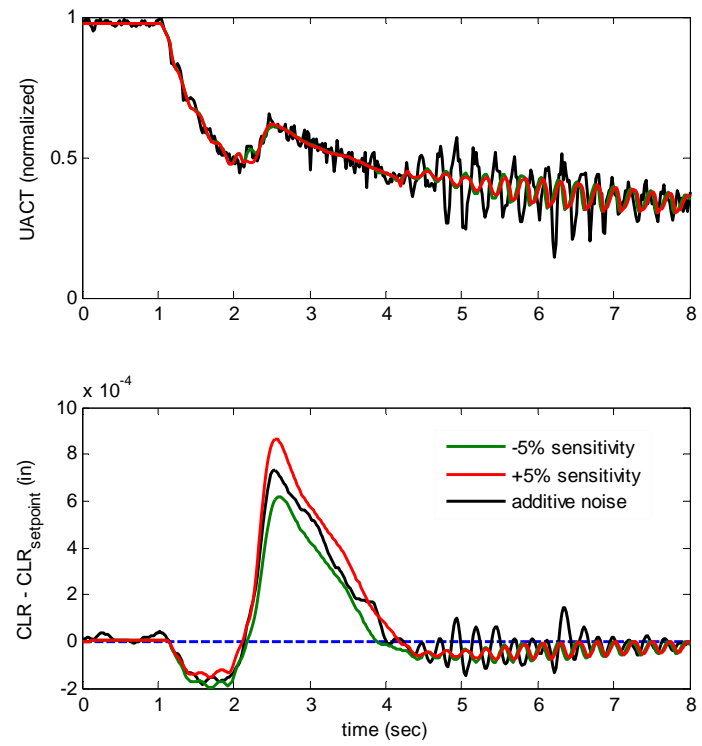

Figure 11. Constrained R-LPV responses for a thrust reverse transient with corrupted measured disturbance.

even divergent control signal. Nevertheless, these results are important in confirming that R-LPV models are indeed viable for use in MPC even in the presence of noise.

\section{Conclusions}

In this paper, an innovative model predictive control (MPC) strategy using rate-based linear parameter varying (R-LPV) models is introduced. The virtues of the rate-based framework permit state derivatives to be directly specified, resulting in the implicit inclusion of state derivative trim values in the family of trajectories within the neighborhood of linearization equilibrium points. This enhances transient performance by widening the model's valid applicable range without introducing additional linearization points, especially at non-equilibrium. Removal of the explicit trim value information furthermore results in models that exhibit less approximation error, an important attribute of any recursive modeling technique, such as quasi-LPV.

The new MPC strategy is applied to an active clearance control system, where it is highly important to minimize clearance but avoid violating the minimum clearance constraint that protects against detrimental turbine blade rubs. In this work, the active clearance control optimization problem consists only of maintaining a minimal clearance set point, however directly optimizing against performance metrics such as exhaust gas temperature (T5) and fuel flow $(W F)$ is a straightforward extension using this framework. The R-LPV strategy was demonstrated to maintain prediction fidelity and control optimality in transient regimes where the conventional Jacobian LPV method does not. Additionally, the controller was proven to be advantageous for tip clearance control when comparing against an analogous unconstrained, gain-scheduled controller similar to LQG.

With regard to computational complexity, although the R-LPV transformation produces models with state dimensions greater than conventional LPV by the number of controlled inputs and outputs, it is shown that the integrated output states may be merged with the disturbance model states. As such, the internal model states are greater by only the number of inputs, which is usually small in propulsion system applications. Although the new MPC strategy was applied to the rather demanding active clearance control problem with competing objectives, the theoretical groundwork presented here provides sufficient generality to carry over to MPC applications in other nonlinear control problems; among the possibilities being turbofan engine control. Because of the demonstrated advantages this method has over conventional methods, it has great potential for control of aerospace applications where maintaining control optimality during transients is especially critical. 


\section{References}

1 Brunell, B.J., Bitmead, R.R., and Connolloy, A.J., "Nonlinear model predictive control of an aircraft gas turbine engine," Proceedings of the $41^{\text {st }}$ IEEE Conference on Decision and Control, Las Vegas, NV, 2002, pp. 4649-4651.

2 Fuller, J.W., Kumar, A., and Millar, R.C., "Adaptive model based control of aircraft propulsion systems: status and outlook for naval aviation applications," ASME Paper GT2006-90241, May 2006.

3 Maciejowski, J.M., Predictive Control with Constraints, Prentice Hall, Harlow, England, 2002.

4 Lattime, S.B. and Steinetz, B.M., "High-pressure turbine clearance control systems: current practices and future directions," Journal of Propulsion and Power, Vol. 20, No. 2, 2004, pp. 302-311.

5 Wiseman, M.W. and Guo, T.-H., "An investigation of life extending control technologies for gas turbine engines," Proceedings of the American Control Conference, Arlington, VA, 2001, pp. 3706-3707.

6 Geisheimer, J.L., Billington, S.A., Holst, T., and Burgess, D.W., "Performance testing of a microwave tip clearance sensor," AIAA Paper 2005-3987, July 2005.

7 DeCastro, J.A., Melcher, K.J., and Noebe, R.D., "System-level design of a shape memory alloy actuator for active clearance control in the high-pressure turbine," AIAA Paper 2005-3988, July 2005.

8 Heise, S.A. and Maciejowski, J.M., "Model predictive control of a supermaneuverable aircraft," AIAA Paper 96-3768, July 1996.

9 Shamma, J.S. and Cloutier, J.R., "Gain-scheduled missile autopilot design using linear parameter varying transformations," Journal of Guidance, Control, and Dynamics, Vol. 16, No. 2, 1993, pp. 256-263.

${ }^{10}$ Mehra, R.K., Prasanth, R.K., Bennett, R.L., Neckels, D., and Wasikowski, M., "Model predictive control design for XV-15 tilt rotor flight control," AIAA Paper 2001-4331, Aug. 2001.

${ }^{11}$ Leith, D.J. and Leithead, W.E., "Input-output linearization by velocity-based gain-scheduling," International Journal of Control, Vol. 72, No. 3, 1999, pp. 229-246.

12 Stengel, R.F., Optimal Control and Estimation, Dover, New York, 1994.

${ }^{13}$ Pannocchia, G. and Rawlings, J.B., "Disturbance models for offset-free model-predictive control," AIChE Journal, Vol. 49, No. 2, 2003, pp. 426-437.

${ }^{14}$ Badgwell, T.A. and Muske, K.R., "Disturbance model design for linear model predictive control," Proceedings of the American Control Conference, Anchorage AK, 2002, pp. 1621-1626.

${ }^{15}$ Lattime, S.B., Steinetz, B.M., and Robbie, M.G., "Test rig for evaluating active turbine blade tip clearance control concepts," Journal of Propulsion and Power, Vol. 21, No. 3, 2005, pp. 552-563.

${ }^{16}$ DeCastro, J.A. and Melcher, K.J., "A study on the requirements for fast active turbine tip clearance control systems," NASA TM-2004-213121, July 2004.

${ }^{17}$ Doyle, J.C. and Stein G., "Robustness with observers," IEEE Transactions on Automatic Control, Vol. AC-24, No. 4, 1979, pp. 607-611.

${ }^{18}$ Olsson, W.J. and Martin R.L., "B747/JT9D flight loads and their effect on engine running clearance and performance deterioration; nacelle aerodynamic and inertial loads (NAIL)/ JT9D jet engine diagnostics programs," NASA CR-165573, Feb. 1982.

${ }^{19}$ Sallee, G.P., "Performance deterioration based on existing (historical) data; JT9D jet engine diagnostics program," NASA CR135448, April 1978. 


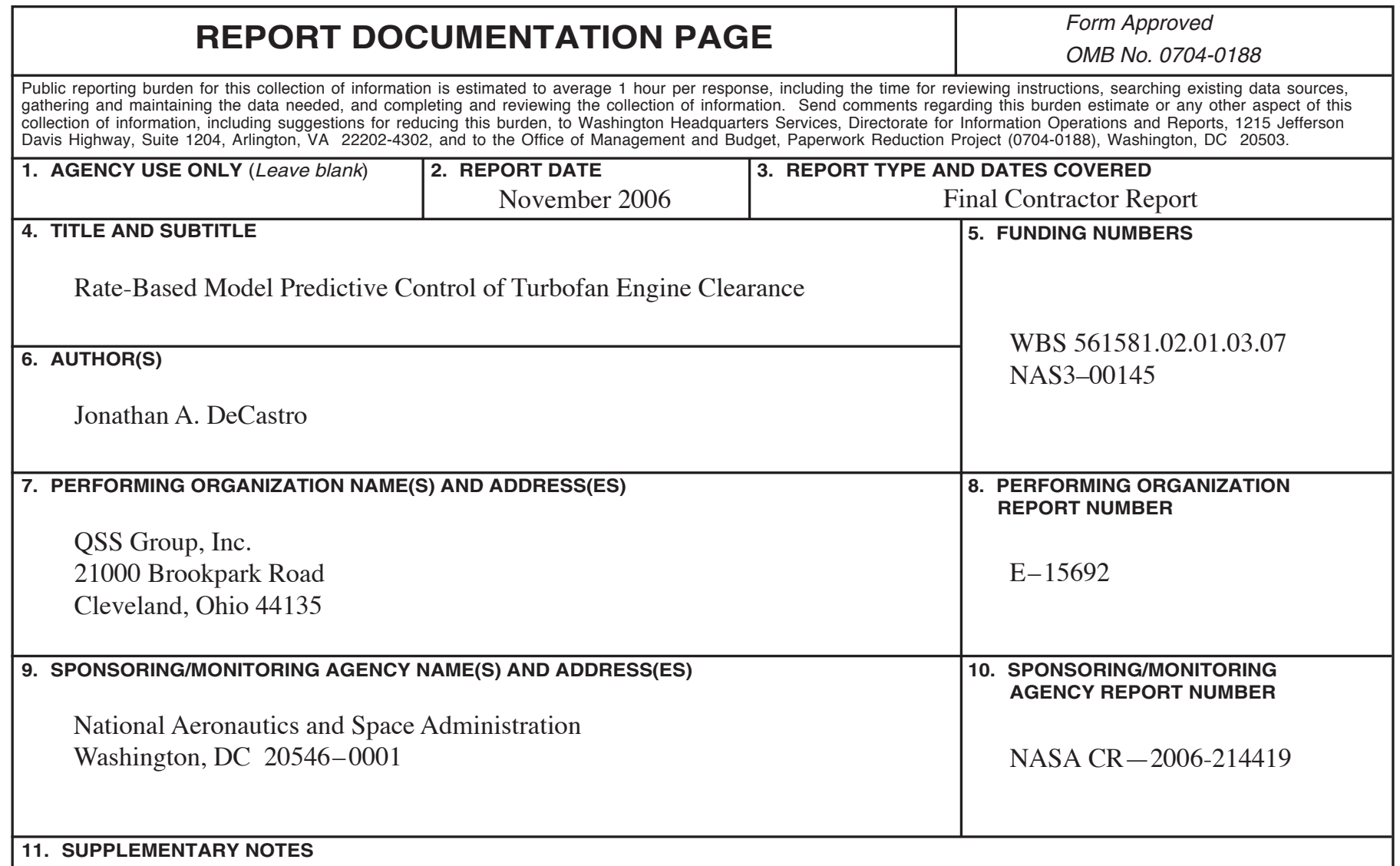

Prepared for the 42nd Joint Propulsion Conference and Exhibit cosponsored by the AIAA, ASME, SAE, and ASEE, Sacramento, California, July 9-12, 2006. Project manager, Ten-Huei Guo, Instrumentation and Controls Division, NASA Glenn Research Center, organization code RIC, 216-433-3734.

\begin{tabular}{|l|l|l|l}
\hline 12a. DISTRIBUTION/AVAILABILITY STATEMENT & 12b. DISTRIBUTION CODE
\end{tabular}

Unclassified - Unlimited

Subject Category: 07

Available electronically at http://gltrs.grc.nasa.gov

This publication is available from the NASA Center for AeroSpace Information, 301-621-0390.

13. ABSTRACT (Maximum 200 words)

An innovative model predictive control strategy is developed for control of nonlinear aircraft propulsion systems and sub-systems. At the heart of the controller is a rate-based linear parameter-varying model that propagates the state derivatives across the prediction horizon, extending prediction fidelity to transient regimes where conventional models begin to lose validity. The new control law is applied to a demanding active clearance control application, where the objectives are to tightly regulate blade tip clearances and also anticipate and avoid detrimental blade-shroud rub occurrences by optimally maintaining a predefined minimum clearance. Simulation results verify that the rate-based controller is capable of satisfying the objectives during realistic flight scenarios where both a conventional Jacobian-based model predictive control law and an unconstrained linear-quadratic optimal controller are incapable of doing so. The controller is evaluated using a variety of different actuators, illustrating the efficacy and versatility of the control approach. It is concluded that the new strategy has promise for this and other nonlinear aerospace applications that place high importance on the attainment of control objectives during transient regimes.

14. SUBJECT TERMS

Optimal control; Adaptive control; Clearances

\begin{tabular}{|c|c|c|}
\hline $\begin{array}{c}\text { 17. SECURITY CLASSIFICATION } \\
\text { OF REPORT } \\
\text { Unclassified }\end{array}$ & $\begin{array}{c}\text { 18. SECURITY CLASSIFICATION } \\
\text { OF THIS PAGE } \\
\text { Unclassified }\end{array}$ & $\begin{array}{c}\text { 19. SECURITY CLASSIFICATION } \\
\text { OF ABSTRACT } \\
\text { Unclassified }\end{array}$ \\
\hline
\end{tabular}

NSN 7540-01-280-5500

Standard Form 298 (Rev. 2-89)

Prescribed by ANSI Std. Z39-18 298-102 

